\title{
Durability and clinical impact of tricuspid valve procedures in patients receiving a continuous-flow left ventricular assist device
}

\author{
Jiho Han, BS, ${ }^{\text {a }}$ Koji Takeda, MD, PhD, ${ }^{\text {a }}$ Hiroo Takayama, MD, PhD, ${ }^{\text {a }}$ Paul A. Kurlansky, MD, \\ Christine M. Mauro, PhD, ${ }^{\mathrm{c}}$ Paolo C. Colombo, MD, ${ }^{\mathrm{b}}$ Melana Yuzefpolskaya, MD, \\ Shinichi Fukuhara, MD, ${ }^{a}$ Lauren K. Truby, MD, ${ }^{a}$ Veli K. Topkara, MD, ${ }^{b}$ Arthur R. Garan, MD, \\ Donna M. Mancini, MD, ${ }^{b}$ and Yoshifumi Naka, MD, $\mathrm{PhD}^{\mathrm{a}}$
}

\begin{abstract}
Objective: Patients evaluated for a continuous-flow left ventricular assist device frequently present with severe right ventricular dysfunction with tricuspid regurgitation. Long-term outcomes of concurrent tricuspid valve procedures in continuous-flow left ventricular assist device implantation are unclear.
\end{abstract}

Methods: From May 2004 to December 2013, 336 patients received continuous-flow left ventricular assist devices. Among these, 8 patients with prior tricuspid valve procedures were excluded. At continuous-flow left ventricular assist device implantation, 76 patients underwent tricuspid valve procedures (group A), including 68 repairs and 8 replacements. The remaining 252 patients did not receive concurrent tricuspid valve procedures (group B).

Results: Preoperatively, group A had higher central venous pressure/pulmonary capillary wedge pressure $(P=.032)$, total bilirubin $(P=.009)$, and percentage of moderate or greater tricuspid regurgitation $(98.7 \%$ vs $18.8 \% ; P<.001)$. In group A, cardiopulmonary bypass time $(136 \pm 52.0$ minutes vs $83.9 \pm 38.8$ minutes; $P<.001)$, intraoperative platelet use (13.6 \pm 6.70 units vs $11.7 \pm 5.92$ units; $P=.042)$, and bleeding requiring reoperation $(27.5 \%$ vs $16.7 \% ; P=.046)$ were significantly increased. In-hospital mortality was similar $(10.5 \%$ vs $6.4 \% ; P=.22)$. On-device 2 -year survival was $73.9 \%$ in group A and $74.2 \%$ in group B $(P=.24)$. At 2 years, mean cumulative readmissions for right heart failure was 0.21 in group $A$ and 0.27 in group B $(P=.95)$. A generalized linear mixed-effects model showed that tricuspid valve procedures are protective for developing future significant tricuspid regurgitation (odds ratio, $0.38 ; 95 \%$ confidence interval, $0.19-0.76 ; P=.006$ ).

Conclusions: Concomitant tricuspid valve procedures at continuous-flow left ventricular assist device implantation can be performed safely and are protective against worsening tricuspid regurgitation during the first 2 years of support. ( $\mathrm{J}$ Thorac Cardiovasc Surg 2016;151:520-527)

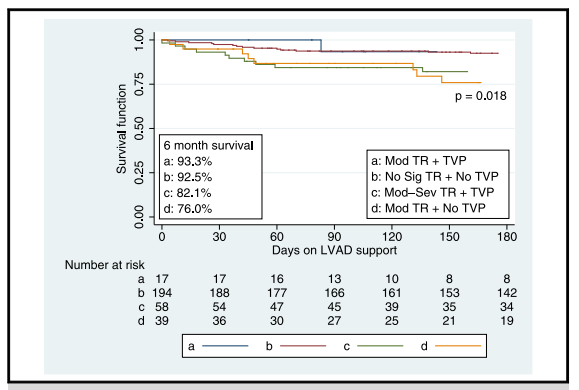

Six-month survival of subgroups based on TVP and degree of preoperative TR.

\section{Central Message}

Patients with CF-LVADs and significant TR should be considered for TVPs at the time of implantation.

\section{Perspective}

No established guidelines exist on the surgical approach of patients with CF-LVADs who present with significant TR. Previous studies were limited in sample size and follow-up length. This study shows long-term safety, durability, and benefits of concomitant TVP performed at a single high-volume center. The results will allow better patient selection and identification of risks and benefits of concurrent TVP.

See Editorial Commentary page 528.
The continuous-flow left ventricular assist device (CF-LVAD) has become the standard of care in patients with advanced heart failure as a bridge to transplantation or destination therapy. ${ }^{1}$

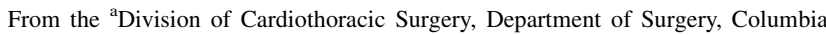
University Medical Center, New York, NY; ${ }^{\mathrm{b}}$ Division of Cardiology, Department of Medicine, Columbia University Medical Center, New York, NY; and ${ }^{\mathrm{c}}$ Department of Biostatistics, Columbia University Mailman School of Public Health, New York, NY.

Received for publication April 25, 2015; revisions received Aug 29, 2015; accepted for publication Sept 11, 2015

Address for reprints: Yoshifumi Naka, MD, PhD, 177 Fort Washington Ave, New York, NY 10032 (E-mail: yn33@ columbia.edu). 0022-5223/\$36.00

Copyright $₫ 2016$ Published by Elsevier Inc. on behalf of The American Association for Thoracic Surgery

http://dx.doi.org/10.1016/j.jtcvs.2015.09.082
Patients referred for CF-LVAD implantation frequently present with biventricular dysfunction, and some have severe right ventricular (RV) dysfunction with tricuspid regurgitation (TR). In these patients, right heart failure (RHF) can persist postoperatively and is associated with worse mortality and morbidity. In an attempt to reduce postoperative RHF, some surgeons perform concomitant tricuspid valve procedures (TVPs), either annuloplasty or replacement, at the time of

Scanning this QR code will take you to supplemental tables for this article. 


\section{Abbreviations and Acronyms \\ CF-LVAD $=$ continuous-flow left ventricular assist device \\ CI $=$ confidence interval \\ $\mathrm{CPB}=$ cardiopulmonary bypass \\ $\mathrm{CVP}=$ central venous pressure \\ HR = hazard ratio \\ IQR $=$ interquartile range \\ LVAD $=$ left ventricular assist device \\ RHF = right heart failure \\ $\mathrm{RV} \quad=$ right ventricle, right ventricular \\ RVAD = right ventricular assist device \\ TR = tricuspid regurgitation \\ TVP $=$ tricuspid valve procedure}

CF-LVAD implantation. ${ }^{2-6}$ Others suggest that CF-LVAD implantation alone will improve right-sided hemodynamics, making TVP unnecessary. ${ }^{7}$ A recent analysis of the Society of Thoracic Surgeons database warned against routine application of concomitant tricuspid procedures because of increased perioperative risks. ${ }^{3}$ However, at this time there are no established guidelines as to which patients should receive concomitant TVP, and many published studies are limited by small sample size and duration of follow-up. In the current era, although more patients are on CF-LVAD support for prolonged periods, it is unclear whether concomitant TVP is beneficial in the long-term. The aim of this study is to evaluate the long-term durability and clinical outcomes of concomitant TVP during CF-LVAD support.

\section{MATERIALS AND METHODS \\ Patient Selection}

The Columbia University Medical Center Institutional Review Board approved this study. A total of 336 patients who received CF-LVADs between April 2004 and December 2013 at Columbia University Medical Center were retrospectively reviewed. Among those, 328 patients who did not undergo previous tricuspid valve surgery were included in this study. At the time of device implantation, TVP was performed in 76 patients $(23.2 \%)$ (group A), including 68 repairs and 8 replacements. The remaining 252 patients $(76.8 \%$ ) did not undergo concurrent TVP (group B).

\section{Devices Used}

Most patients $(\mathrm{n}=276 ; 84.2 \%)$ received the HeartMate II device (Thoratec Corp, Pleasanton, Calif). Other left ventricular assist devices (LVADs) implanted included $29(8.8 \%)$ HeartWare HVADs (HeartWare International, Inc, Framingham, Mass), 9 (2.7\%) VentrAssist devices (Ventracor Ltd, Chatswood, NSW, Australia), 8 (2.4\%) DuraHeart Left Ventricular Assist Systems (Terumo Heart, Ann Arbor, Mich), and 6 (1.8\%) DeBakey Ventricular Assist Devices (MicroMed Technology, Inc, Houston, Tex).

\section{Surgical Indication and Procedures for Tricuspid Valve Regurgitation}

The decision to perform TVP was made on the basis of severity of TR by intraoperative transesophageal echocardiography. In general, TVP was performed for moderate or greater TR. Tricuspid valve annuloplasty with an annuloplasty ring was the first choice of procedure. In the case of severe leaflet tethering or destruction, tricuspid replacement was performed with a bioprosthetic valve.

\section{Other Concomitant Valve Procedures}

In patients with mild or greater aortic insufficiency, aortic valve repair or replacement with a tissue valve was performed. In most cases, the aortic valve was repaired by approximating the raphe of each leaflet. Patients with a mechanical valve in the aortic position had the aortic valve oversewn with a patch. Mitral valve repair was performed in patients with severe functional mitral regurgitation at the discretion of the surgeon.

\section{Postimplant Device Management}

After device implantation, all patients received a standardized heart failure medical regimen including neurohormonal antagonists, diuretics, and antiarrhythmic agents if needed on the basis of the individual clinical picture. Heparin was bridged on the basis of the Columbia University Medical Center's protocol. Anticoagulation therapy with aspirin and warfarin is implemented once adequate hemostasis has been achieved. Patients received follow-up at 1 week after the initial discharge and monthly thereafter unless an issue necessitated more frequent visits. Clinic visit frequency varied among patients depending on individual medical issues and travel distances.

\section{Data Collection}

Patient data were obtained from electronic medical records. Primary outcome variables included 2-year on-device survival, freedom from RHF readmission, and degree of TR on serial echocardiography. Echocardiography results were obtained immediately before CF-LVAD implantation, during the operation, and at 30 days, 6 months, 1 year, and 2 years of support. Severity of TR was divided into none, mild, mild-moderate, moderate, moderate-severe, or severe. Significant TR was defined as moderate or greater TR at any point in the follow-up period. Pre-LVAD assessment of RV function was binary (severe or not severe) because of the lack of universal consensus of classification system on RV function and difficulty of implementing any of existing classification system retrospectively. Patients were censored at the time of orthotopic heart transplant or device removal after recovery of the native heart. Preoperative variables that may affect the primary outcome were identified, including demographics, medical histories, laboratory values, and hemodynamics. Intraoperative variables, such as cardiopulmonary bypass (CPB) time, aortic crossclamp time, use of blood products, and concomitant cardiac procedures, were also collected. Early postimplant data included complications occurring between the operation and the hospital discharge. Major adverse events requiring readmission during CFLVAD support were also recorded. These included RHF, major bleeding events such as gastrointestinal tract bleeding and significant epistaxis, device-related events such as pump thrombi and driveline injury, major cerebral events, cardiac arrhythmia, and infections related or not related to the device. Because there is no consensus on the definition of RHF, it was based on clinical presentation, including jugular venous distension, peripheral edema, and ascites. History and physical examination were performed by heart failure cardiologists. Heart failure due to device malfunction (including thrombosis, obstruction, and driveline fractures) was excluded. The records were retrospectively reviewed by authors KT and HT. Followup data were collected in 299 patients $(91.1 \%)$ for a period between 5 and 3091 days (median, 735 days; interquartile range [IQR], 363-1266 days). Follow-up was complete as of August 1, 2014.

\section{Statistical Analysis}

Stata 12 software (StataCorp LP, College Station, Tex) and SAS 9.4 (SAS Institute Inc, Cary, NC) were used in statistical analyses. Statistical significance was determined on the basis of predetermined $\alpha=0.05$. Categoric variables were summarized with frequencies and percentages 
TABLE 1. Preoperative baseline characteristics

\begin{tabular}{|c|c|c|c|c|}
\hline Variables & $\begin{array}{c}\text { All } \\
\mathbf{N}=\mathbf{3 2 8}\end{array}$ & $\begin{array}{c}\text { Group A } \\
\mathbf{N}=76\end{array}$ & $\begin{array}{l}\text { Group B } \\
\mathbf{N}=\mathbf{2 5 2}\end{array}$ & $P$ value \\
\hline Age of LVAD implant (y) & $57 \pm 14$ & $57 \pm 13$ & $57 \pm 14$ & .78 \\
\hline $\mathrm{BMI}$, mean $\pm \mathrm{SD}$ & $26.2 \pm 5.71$ & $26.5 \pm 5.64$ & $26.1 \pm 5.74$ & .57 \\
\hline $\mathrm{BSA}\left(\mathrm{m}^{2}\right)$, mean $\pm \mathrm{SD}$ & $1.95 \pm 0.25$ & $1.99 \pm 0.27$ & $1.94 \pm 0.24$ & .08 \\
\hline Gender, male, n (\%) & $269(82.0)$ & $58(76.3)$ & $211(83.7)$ & .14 \\
\hline Hypertension, n (\%) & $158(48.2)$ & $36(47.4)$ & $122(48.4)$ & .87 \\
\hline Diabetes mellitus, n (\%) & $111(33.8)$ & $23(30.3)$ & $88(34.9)$ & .45 \\
\hline Hyperlipidemia, n (\%) & $116(35.4)$ & $23(30.3)$ & $93(36.9)$ & .29 \\
\hline Cause of heart failure, n (\%) & & & & .21 \\
\hline Nonischemic DCM & 163 (49.7) & $44(57.9)$ & $119(47.2)$ & \\
\hline Ischemic cardiomyopathy & $137(41.8)$ & $28(36.8)$ & $109(43.3)$ & \\
\hline Others & $28(8.5)$ & $4(5.3)$ & $24(9.5)$ & \\
\hline $\mathrm{ICD}, \mathrm{n}(\%)$ & $262(80.1)$ & $66(86.8)$ & $196(78.1)$ & .09 \\
\hline Redo sternotomy (\%) & $113(34.5)$ & $28(36.8)$ & 85 (33.7) & .68 \\
\hline Preoperative inotrope support & $276(84.2)$ & $68(89.5)$ & $208(82.5)$ & .15 \\
\hline Preoperative IABP support, n (\%) & $87(26.7)$ & $25(32.9)$ & $62(24.8)$ & .16 \\
\hline Preoperative vasopressors, n (\%) & $32(10.2)$ & $10(13.2)$ & $22(9.2)$ & .33 \\
\hline Preoperative ventilator use, $\mathrm{n}(\%)$ & $15(4.8)$ & $3(4.0)$ & $12(5.0)$ & .71 \\
\hline \multicolumn{5}{|l|}{ Preoperative MCS use, n (\%) } \\
\hline ECMO & $10(3.1)$ & $2(2.6)$ & $8(3.2)$ & .81 \\
\hline Impella (Abiomed Inc, Danvers, Mass) & $4(1.2)$ & $1(1.3)$ & $3(1.2)$ & .93 \\
\hline CentriMag VAD (Thoratec Corp, Pleasanton, Calif) & $24(7.3)$ & $4(5.3)$ & $20(7.9)$ & .43 \\
\hline $\operatorname{LVEF}(\%)$ & $15.1 \pm 6.21$ & $14.4 \pm 5.93$ & $15.3 \pm 6.29$ & .27 \\
\hline Severe RV dysfunction, n (\%) & $81(28.7)$ & $23(37.1)$ & $58(26.3)$ & .11 \\
\hline TR grade, $\mathrm{n}(\%)$ & & & & $<.001$ \\
\hline None-trace & $70(22.2)$ & $0(0.0)$ & $70(29.3)$ & \\
\hline Mild & $113(35.9)$ & $1(1.3)$ & $112(46.9)$ & \\
\hline Mild-moderate & $12(3.8)$ & $0(0.0)$ & $12(5.0)$ & \\
\hline Significant TR & $120(38.1)$ & $75(98.7)$ & $45(18.8)$ & \\
\hline Moderate & $56(17.8)$ & $17(22.4)$ & $39(16.3)$ & \\
\hline Moderate-severe & $16(5.1)$ & $10(13.2)$ & $6(2.5)$ & \\
\hline Severe & $48(15.2)$ & $48(63.2)$ & $0(0)$ & \\
\hline CVP (mm Hg) & $11.0 \pm 5.48$ & $12.1 \pm 5.98$ & $10.7 \pm 5.29$ & .054 \\
\hline Mean PAP (mm Hg) & $34.8 \pm 9.53$ & $34.4 \pm 8.14$ & $35.0 \pm 9.94$ & .66 \\
\hline PCWP (mm Hg) & $23.8 \pm 7.95$ & $24.0 \pm 7.27$ & $23.8 \pm 8.16$ & .86 \\
\hline CVP/PCWP & $0.494 \pm 0.265$ & $0.556 \pm 0.279$ & $0.475 \pm 0.258$ & .032 \\
\hline $\mathrm{CO}(\mathrm{L} / \mathrm{min})$ & $3.32 \pm 1.03$ & $3.19 \pm 1.04$ & $3.35 \pm 1.03$ & .29 \\
\hline PVR (Wood units) & $3.91 \pm 2.55$ & $3.70 \pm 1.95$ & $3.97 \pm 2.70$ & .48 \\
\hline $\mathrm{WBC}(\times 1000 / \mathrm{mL})$ & $8.66 \pm 3.21$ & $8.15 \pm 2.46$ & $8.82 \pm 3.39$ & .11 \\
\hline Hemoglobin $(\mathrm{g} / \mathrm{dL})$ & $11.3 \pm 2.03$ & $10.8 \pm 1.84$ & $11.5 \pm 2.06$ & .008 \\
\hline Hematocrit $(\%)$ & $34.7 \pm 5.80$ & $33.3 \pm 5.55$ & $35.1 \pm 5.82$ & .017 \\
\hline Platelets $(\times 1000 / \mathrm{mL})$ & $210 \pm 74.0$ & $211 \pm 83.9$ & $209 \pm 71.2$ & .88 \\
\hline Sodium (mmol/L) & $134 \pm 4.50$ & $133 \pm 4.66$ & $134 \pm 4.43$ & .086 \\
\hline BUN (mg/dL) & $35.2 \pm 19.6$ & $35.9 \pm 17.1$ & $35.0 \pm 20.3$ & .72 \\
\hline Creatine (mg/dL) & $1.52 \pm 0.722$ & $1.50 \pm 0.690$ & $1.52 \pm 0.733$ & .82 \\
\hline Albumin $(\mathrm{g} / \mathrm{dL})$ & $3.59 \pm 0.540$ & $3.52 \pm 0.557$ & $3.61 \pm 0.534$ & .23 \\
\hline AST (IU/L) & $23.5(18.0-35.5)$ & $23.5(18.0-37.5)$ & $23.5(18.0-35.0)$ & .17 \\
\hline ALT (IU/L) & $22.0(15.0-42.5)$ & $20.0(12.5-42.5)$ & $23.0(15.0-41.5)$ & .29 \\
\hline Total bilirubin (mg/dL) & $1.50 \pm 1.17$ & $1.80 \pm 1.36$ & $1.40 \pm 1.09$ & .009 \\
\hline INR & $1.38 \pm 0.403$ & $1.36 \pm 0.306$ & $1.38 \pm 0.428$ & .59 \\
\hline
\end{tabular}

Data are expressed as mean \pm standard deviation or n (\%) or median (interquartile range). Differences were considered significant when $P<.05$, and are listed in bold. $L V A D$, Left ventricular assist device; $B M I$, body mass index; $S D$, standard deviation; $B S A$, body surface area; $D C M$, dilated cardiomyopathy; $I C D$, implantable cardioverter-defibrillator; $I A B P$, intra-aortic balloon pump; $M C S$, mechanical circulatory support; $E C M O$, extracorporeal membrane oxygenation; VAD, ventricular assist device; $L V E F$, left ventricular ejection fraction; $R V$, right ventricle; $T R$, tricuspid regurgitation; $C V P$, central venous pressure; $P A P$, pulmonary artery pressure; $P C W P$, pulmonary capillary wedge pressure; $C O$, cardiac output; $P V R$, peripheral vascular resistance; $W B C$, white blood cells; $B U N$, blood urea nitrogen; $A S T$, aspartate aminotransferase; $A L T$, alanine aminotransferase; $I N R$, international normalized ratio. 
and compared across groups using chi-square or Fisher exact tests. Continuous variables are summarized as mean \pm standard deviation, and 2 -sample $t$ tests were used for comparisons across groups. Survival was estimated using Kaplan-Meier curves and compared across groups using log-rank tests. Cox regression models for survival outcomes were used to adjust for the effects of preoperative variables on survival. Because readmissions are repeating events after index discharge, Nelson's ${ }^{8}$ nonparametric method was used to estimate the mean cumulative function for readmission for each cause by TVP status. To test for equality of the mean cumulative function based on TVP, chi-square tests for equality of mean functions were performed. All plots were truncated after 730 days ( 2 years) of observation because of limited observations at later time points. For longitudinal observations, a generalized linear mixed-effects model was used with a random intercept for each person to account for correlations. ${ }^{9}$

\section{RESULTS}

\section{Baseline Characteristics}

Table 1 outlines the preoperative baseline characteristics of the 2 groups. The mean age of the entire cohort was $57 \pm 14$ years, 269 patients $(82 \%)$ were male, 85 patients $(25.9 \%)$ had destination therapy, and 137 patients $(41.8 \%)$ had ischemic cause. Demographics such as age, body mass index, and gender showed no difference between the groups. Group A had lower hemoglobin levels and hematocrit, and higher central venous pressure (CVP), CVP/pulmonary capillary wedge pressure ratio, total bilirubin, and percentage of significant TR than group B (Table 1). Immediately before CF-LVAD implantation, group A had increased RV dysfunction as defined by severe grade in preoperative transthoracic echocardiography $(37.1 \%$ vs $26.3 \% ; P=.11)$.

\section{Intraoperative and Early Postoperative Outcomes}

Intraoperative and early postoperative outcomes are shown in Tables 2 and 3. CPB time was significantly elevated in group A compared with group B, whereas ischemic time showed no difference. Group A used more platelets compared with group B, and rates of bleeding requiring reoperation were significantly increased. At the time of operation, 149 patients $(45.4 \%)$ received concomitant procedures other than TVP. In group A, aortic and mitral valve repairs were performed in 12 patients $(17.1 \%)$ and 14 patients $(18.4 \%)$, respectively, and incidences were similar in group B $(P=.88$ and .35 , respectively). Overall hospital stay and in-hospital mortality were similar between the groups.

\section{Right Ventricular Assist Device Use}

In the entire cohort, a right ventricular assist device (RVAD) was used in 26 patients $(7.9 \%) ; 20(6.1 \%)$ were placed within 14 days after implantation, and 13 were placed within the initial 24 hours of LVAD implantation. The CentriMag device (Thoratec Corp) was used as a temporary RVAD. Median support duration was 14 days (IQR, 10.3-20.8 days), and median postoperative time of RVAD implant was day 0 (IQR, 0-10.5 days). While on RVAD support, 11 patients $(42.3 \%)$ died, 11 patients $(42.3 \%)$ were weaned successfully, 3 patients $(11.5 \%)$
TABLE 2. Intraoperative characteristics

\begin{tabular}{|c|c|c|c|c|}
\hline Variables & $\begin{array}{l}\text { All patients } \\
\mathbf{N}=\mathbf{3 2 8}\end{array}$ & $\begin{array}{c}\text { Group A } \\
\mathbf{N}=76 \\
\end{array}$ & $\begin{array}{l}\text { Group B } \\
\mathbf{N}=\mathbf{2 5 2}\end{array}$ & $\begin{array}{c}P \\
\text { value } \\
\end{array}$ \\
\hline On-pump surgery, n ( $\%)$ & $317(98.5)$ & $75(100)$ & $242(98.0)$ & .21 \\
\hline $\begin{array}{l}\mathrm{CPB} \text { time }(\min ) \\
\quad \text { mean } \pm \mathrm{SD}\end{array}$ & $96.3 \pm 47.8$ & $136 \pm 52.0$ & $83.9 \pm 38.8$ & $<.0001$ \\
\hline Crossclamp used, n (\%) & $74(22.6)$ & $17(22.4)$ & $57(22.6)$ & .96 \\
\hline $\begin{array}{l}\text { Crossclamp time }(\min ) \\
\quad \text { mean } \pm \mathrm{SD}\end{array}$ & $28.5 \pm 13.3$ & $29.0 \pm 18.5$ & $28.4 \pm 11.5$ & .86 \\
\hline \multicolumn{5}{|l|}{$\begin{array}{l}\text { Intraoperative blood } \\
\text { products }\end{array}$} \\
\hline PRBC use, n (\%) & $153(48.6)$ & $35(48.6)$ & $118(48.6)$ & .99 \\
\hline $\begin{array}{l}\text { PRBC (units) } \\
\text { mean } \pm \mathrm{SD}\end{array}$ & $3.49 \pm 2.38$ & $3.51 \pm 2.39$ & $3.48 \pm 2.39$ & .95 \\
\hline FFP use, n $(\%)$ & $232(73.4)$ & $55(75.3)$ & $177(72.8)$ & .67 \\
\hline $\begin{array}{l}\text { FFP (units) } \\
\quad \text { mean } \pm \text { SD }\end{array}$ & $4.14 \pm 2.52$ & $4.38 \pm 2.52$ & $4.06 \pm 2.52$ & .41 \\
\hline Platelets use, n (\%) & $256(81.0)$ & $60(81.0)$ & $196(80.7)$ & .77 \\
\hline $\begin{array}{l}\text { Platelets (units), } \\
\quad \text { mean } \pm \mathrm{SD}\end{array}$ & $12.2 \pm 6.15$ & $13.6 \pm 6.70$ & $11.7 \pm 5.92$ & .042 \\
\hline LVAD indication & & & & .42 \\
\hline BTT, n $(\%)$ & $243(74.1)$ & $59(77.6)$ & $184(73.0)$ & \\
\hline DT, n $(\%)$ & 85 (25.9) & $17(22.4)$ & $68(27.0)$ & \\
\hline LVAD, n (\%) & & & & .78 \\
\hline $\begin{array}{l}\text { HeartMate II } \\
\text { (Thoratec Corp) }\end{array}$ & $276(84.2)$ & $67(88.2)$ & $209(82.9)$ & \\
\hline $\begin{array}{c}\text { HeartWare (HeartWare } \\
\text { International, Inc, } \\
\text { Framingham, Mass) }\end{array}$ & $29(8.8)$ & $4(5.3)$ & $25(9.9)$ & \\
\hline $\begin{array}{c}\text { DeBakey (MicroMed } \\
\text { Technology, Inc, } \\
\text { Houston, Tex) }\end{array}$ & $6(1.8)$ & $1(1.3)$ & $5(2.0)$ & \\
\hline $\begin{array}{c}\text { VentrAssist (Ventracor } \\
\text { Ltd, Chatswood, } \\
\text { NSW, Australia) }\end{array}$ & $9(2.7)$ & $2(2.6)$ & $7(2.8)$ & \\
\hline $\begin{array}{l}\text { DuraHeart (Terumo } \\
\text { Heart, Ann Arbor, } \\
\text { Mich) }\end{array}$ & $8(2.4)$ & $2(2.6)$ & $6(2.4)$ & \\
\hline $\begin{array}{l}\text { Concomitant surgery, } \\
\text { n }(\%)\end{array}$ & $149(45.4)$ & $35(46.1)$ & $114(45.2)$ & .90 \\
\hline AVP/AVR & $58(17.7)$ & $12(17.1)$ & $46(17.9)$ & .88 \\
\hline MVP & 49 (14.9) & $14(18.4)$ & 35 (13.9) & .35 \\
\hline PFO closure & $18(5.5)$ & $5(6.6)$ & $13(5.2)$ & .63 \\
\hline RVAD placement & $15(4.6)$ & $4(5.3)$ & $11(4.4)$ & .72 \\
\hline ECMO placement & $3(0.9)$ & $2(2.6)$ & $1(0.4)$ & .07 \\
\hline MCS removal & $30(9.2)$ & $6(7.9)$ & $25(9.5)$ & .67 \\
\hline Other & $21(6.4)$ & $7(9.2)$ & $14(5.6)$ & .25 \\
\hline
\end{tabular}

Data are expressed as mean \pm standard deviation or $\mathrm{n}(\%)$. Differences were considered significant when $P<.05$, and are listed in bold. TVP, Tricuspid valve procedure; $C P B$, cardiopulmonary bypass; $S D$, standard deviation; $P R B C$, packed red blood cells; FFP, fresh-frozen plasma; $L V A D$, left ventricular assist device; $B T T$, bridge to transplantation; $D T$, destination therapy; $A V P$, aortic valvuloplasty; $A V R$, aortic replacement; $M V P$, mitral valvuloplasty; $P F O$, patent foramen ovale; $R V A D$, right ventricular assist device; $E C M O$, extracorporeal membrane oxygenation; $M C S$, mechanical circulatory support.

underwent transplantation, and 1 patient $(3.8 \%)$ received a HeartWare HVAD (HeartWare International, Inc) as a durable RVAD. Group A had a nonsignificant increase in 
TABLE 3. Early and late mortality and morbidity

\begin{tabular}{|c|c|c|c|c|}
\hline Variables & $\begin{array}{l}\text { All patients } \\
\mathbf{N}=\mathbf{3 2 8}\end{array}$ & $\begin{array}{c}\text { Group A } \\
\mathbf{N}=76\end{array}$ & $\begin{array}{l}\text { Group B } \\
\mathbf{N}=\mathbf{2 5 2}\end{array}$ & $\begin{array}{c}P \\
\text { value }\end{array}$ \\
\hline ICU stay $(d)$, mean $\pm S D$ & $10.2 \pm 10.5$ & $11.0 \pm 10.2$ & $9.94 \pm 10.6$ & .44 \\
\hline $\begin{array}{l}\text { Hospital stay }(\mathrm{d}) \\
\quad \text { mean } \pm \mathrm{SD}\end{array}$ & $36.3 \pm 36.4$ & $40.6 \pm 50.2$ & $35.0 \pm 31.1$ & .25 \\
\hline 30-d mortality, n (\%) & $12(3.7)$ & $4(5.3)$ & $8(3.2)$ & .40 \\
\hline In-hospital mortality, n (\%) & $24(7.3)$ & $8(10.5)$ & $16(6.4)$ & .22 \\
\hline \multicolumn{5}{|l|}{$\begin{array}{l}\text { Postoperative } \\
\text { complications, n (\%) }\end{array}$} \\
\hline VF/VT & $79(26.3)$ & $23(32.9)$ & $56(24.7)$ & .18 \\
\hline Atrial fibrillation & $80(27.0)$ & $18(26.5)$ & $62(27.2)$ & .91 \\
\hline Sepsis & $25(8.6)$ & $4(6.1)$ & $21(9.3)$ & .41 \\
\hline UTI & $37(12.6)$ & $7(10.5)$ & $30(13.3)$ & .54 \\
\hline CVA & $23(7.1)$ & $5(6.6)$ & $18(7.2)$ & .85 \\
\hline Take-back for bleeding & $57(19.3)$ & $19(27.5)$ & $38(16.7)$ & .046 \\
\hline $\begin{array}{l}\text { Renal failure requiring } \\
\text { dialysis }\end{array}$ & $51(16.0)$ & $14(18.9)$ & $37(15.1)$ & .43 \\
\hline $\begin{array}{l}\text { Readmission (overall), } \\
\text { average cumulative } \\
\text { events at } 2 \text { years }\end{array}$ & 1.72 & 2.16 & 1.60 & .091 \\
\hline Arrhythmia & 0.14 & 0.21 & 0.12 & .12 \\
\hline $\begin{array}{l}\text { Bleeding (including } \\
\text { take-back) }\end{array}$ & 0.72 & 0.98 & 0.64 & .14 \\
\hline Device malfunction & 0.32 & 0.30 & 0.33 & .28 \\
\hline LVAD-related infection & 0.28 & 0.46 & 0.24 & .25 \\
\hline RHF & 0.26 & 0.21 & 0.27 & .95 \\
\hline Device exchange & & & & .33 \\
\hline None & $268(81.7)$ & $58(76.3)$ & $210(83.3)$ & \\
\hline Once & $49(14.9)$ & $14(18.4)$ & 35 (13.9) & \\
\hline Twice & $11(3.4)$ & $4(5.3)$ & $7(2.8)$ & \\
\hline
\end{tabular}

Data are expressed as mean \pm standard deviation or $\mathrm{n}(\%)$. Differences were considered significant when $P<.05$, and are listed in bold. $I C U$, Intensive care unit; $S D$, standard deviation; $V F$, ventricular fibrillation; $V T$, ventricular tachycardia; $U T I$, urinary tract infection; $C V A$, cerebrovascular accident; $L V A D$, left ventricular assist device; $R H F$, right heart failure.

overall RVAD use $(13.2 \%$ vs $6.3 \% ; P=.09)$ and within the first 14 days $(7.9 \%$ vs $5.6 \% ; P=.42)$. In group $\mathrm{A}, 5$ patients died, 3 patients recovered, 1 patient proceeded to transplant, and 1 patient received a durable RVAD. In group B, 6 patients died, 8 patients recovered, and 2 patients underwent transplantation. The outcomes after RVAD implantation did not differ within the 2 groups $(P=.49)$.

\section{Late Mortality and Morbidity}

There were 41 patients who died after indexed discharge. Death occurred at a median of 250 days (IQR, 117-621 days). The most common cause of death was stroke ( $\mathrm{n}=8 ; 19.5 \%)$, followed by battery accidents and device malfunction (both $n=4 ; 9.8 \%$ ). For the entire cohort, the estimated on-device survival was $96.0 \%, 88.2 \%, 82.1 \%$, and $74.1 \%$ at 30 days, 6 months, 1 year, and 2 years, respectively, after initial hospital discharge. At the end of 2 years, groups $\mathrm{A}$ and $\mathrm{B}$ had similar survivals of $73.9 \%$ and $74.2 \%$, respectively $(P=.24$ ) (Figure 1 and Table E1). In the Cox regression model, TVP (hazard ratio

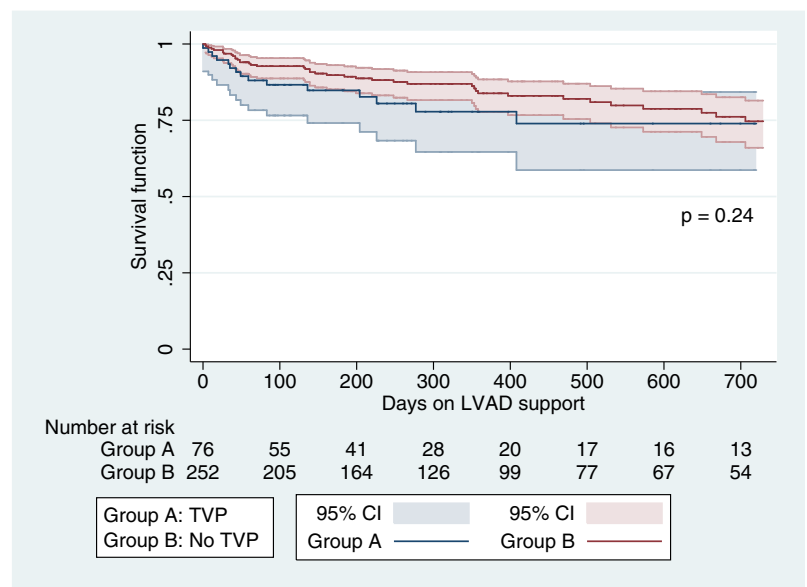

FIGURE 1. Overall 2-year survival in group A versus B. Group A: TVP. Group B: no TVP. LVAD, Left ventricular assist device; TVP, tricuspid valve procedure.

[HR], 0.26; 95\% confidence interval [CI], 0.07 to -0.90 ; $P=.03$ ) was associated with decreased mortality, whereas increase in CPB time (HR, 1.01; 95\% CI, 1.01-1.02; $P<.001)$ and preoperative creatinine (HR, 1.42; 95\% CI, $1.17-1.72 ; P<.001)$ were associated with adverse 2 -year survival. Groups A and B were further divided on the basis of the severity of preoperative TR (no significant TR, moderate TR, and greater than moderate TR in each group). On-device survival of all 4 groups was compared (Figure 2 and Table E2). At 6 months, survival was $93.3 \%$ in patients with moderate TR in group A, 92.5\% in patients with no significant TR in group B, $82.1 \%$ in patients with greater than moderate TR in group $\mathrm{A}$, and $76.0 \%$ in patients with moderate TR in group $\mathrm{B}(P=.018)$. Patients with preoperative moderate TR were divided into 2

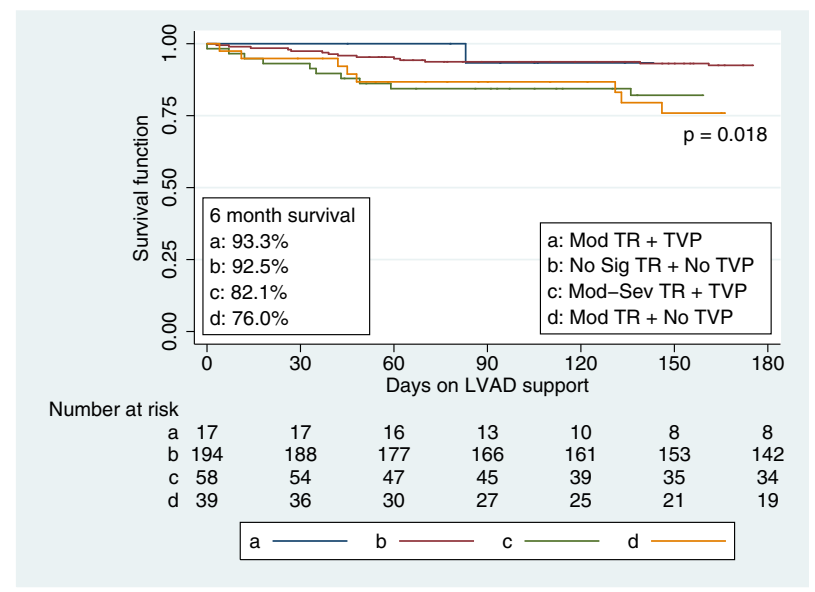

FIGURE 2. Six-month survival of subgroups based on TVP and degree of preoperative TR. A, Moderate TR with TVP. B, No significant TR and no TVP. C, Moderate TR and no TVP. D, Moderate-severe TR with TVP. TR, Tricuspid regurgitation; TVP, tricuspid valve procedure; $L V A D$, left ventricular assist device. 


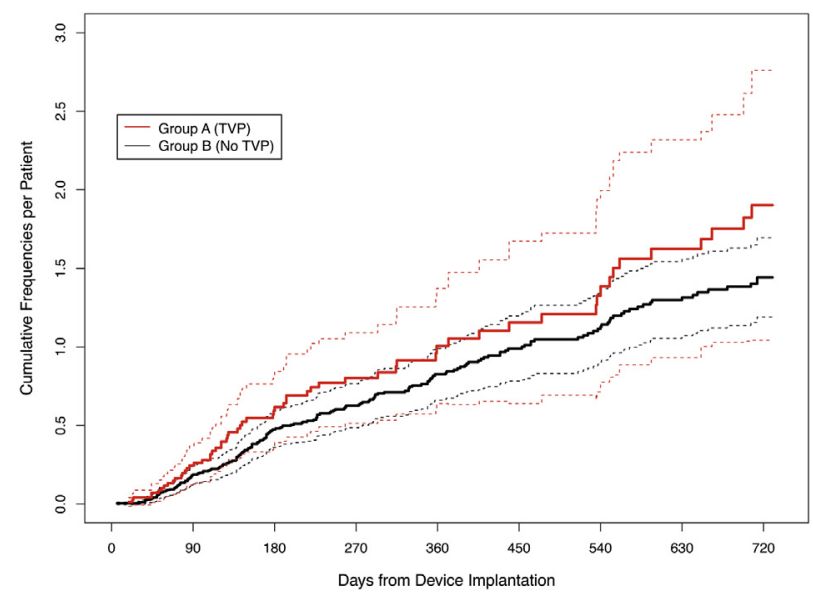

FIGURE 3. Two-year mean cumulative overall readmissions in group A versus B. Group A: TVP. Group B: no TVP. TVP, Tricuspid valve procedure.

subgroups: those who received TVP $(\mathrm{n}=17)$ and those who did not $(\mathrm{n}=39)$. Overall 6-month survival between these 2 groups did not yield any statistically significant difference (graph not shown; $P=.17$ ).

After initial discharge, the mean cumulative number of overall readmissions was 2.16 in group $\mathrm{A}$ and 1.60 in group $\mathrm{B}$ at 2 years (Figure 3) $(P=.09)$. When divided by cause, there was no difference in the mean cumulative number of events (Table 3). Because there was a significant difference in incidences of immediate take-back for bleeding, a separate analysis including these events was performed, and the results showed no significant difference in group A versus $\mathrm{B}(P=.14)$. In group $\mathrm{A}$, mean cumulative readmission due to RHF was 0.21 versus 0.27 in group B at 2 years of support, which was unaffected by TVP (Figure 4) $(P=.95)$.

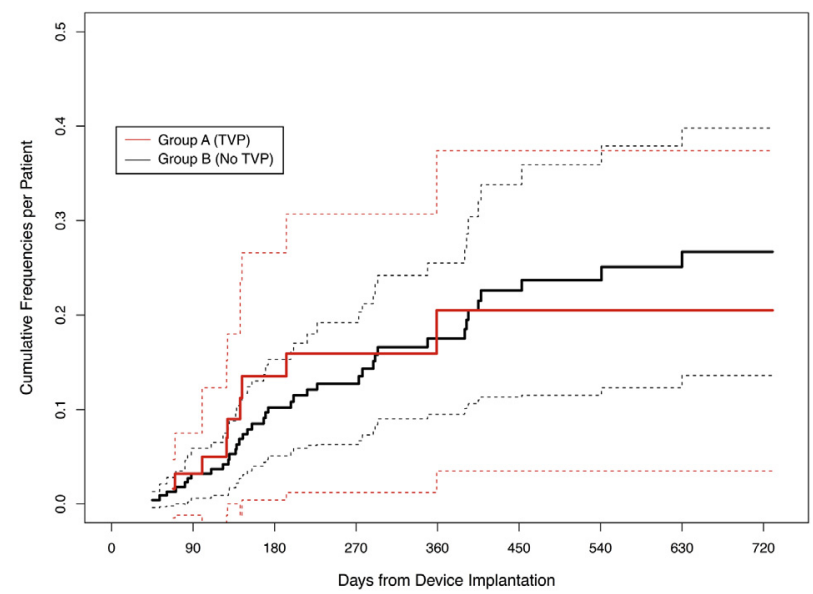

FIGURE 4. Two-year mean cumulative readmissions due to RHF in group A versus B. Group A: TVP. Group B: no TVP. TVP, Tricuspid valve procedure.

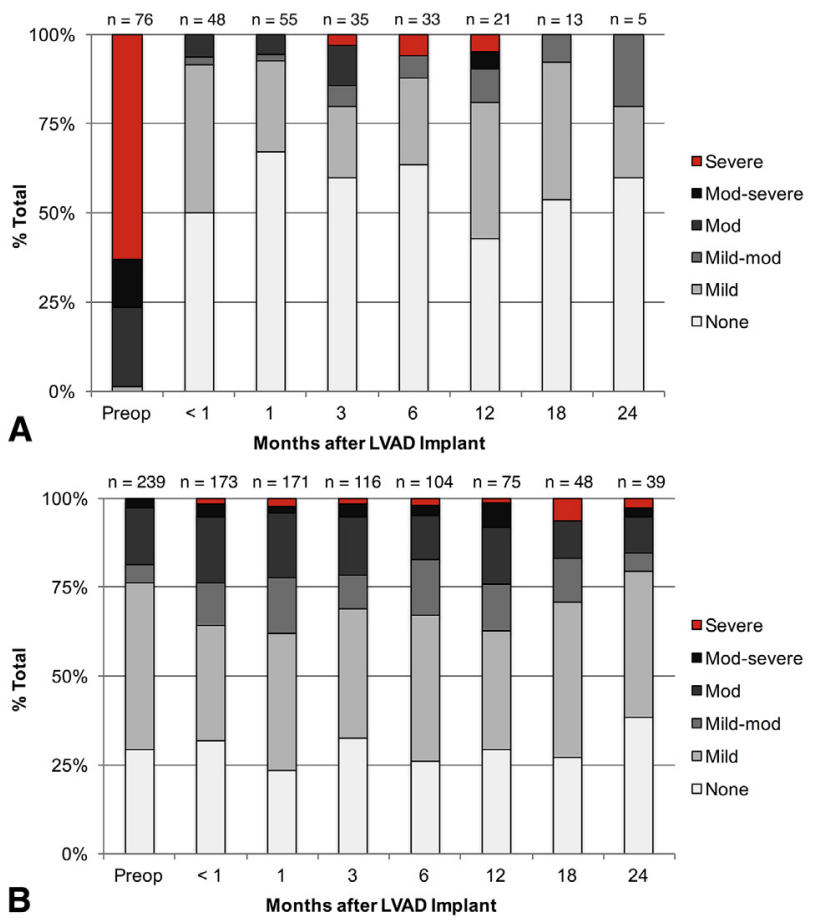

FIGURE 5. A, Frequencies of TR severity over time in group A (TVP). $\mathrm{B}$, Frequencies of TR severity over time in group B (no TVP). LVAD, Left ventricular assist device.

\section{Durability of Tricuspid Valve Procedures}

Of 68 patients who received TV repair, an Edwards MC3 (Edwards Lifesciences, Irvine, Calif) annuloplasty ring was used in 31 patients $(45.6 \%)$. Contour 3D (Medtronic, Minneapolis, Minn) and ATS Simulus (LeviBio Medica, Roma, Italy) annuloplasty rings were used in 18 patients $(26.5 \%)$ and 8 patients $(11.8 \%)$, respectively. The Kay procedure was used in suture annuloplasty $(\mathrm{n}=2 ; 2.9 \%)$. Figure 5, $A$ and $B$ show frequencies of various TR grades over time after initial hospital discharge in patients with TV repair and no repair. Within 1 month after discharge, patients who received TV repair had marked improvement in TR grade. Of 48 patients with echocardiography results, $45(93.8 \%)$ had less than moderate TR and most $(n=44 ; 91.7 \%)$ had none or mild. At 1 month, $94.9 \%$ of patients were free from significant TR, followed by $86.4 \%$ and $83.1 \%$ at 6 and 9 months, respectively (Figure 5, A). Longitudinal analysis using generalized linear mixed-effects model showed that having a TVP is protective for developing future significant TR (odds ratio, $0.38 ; 95 \% \mathrm{CI}, 0.19-0.76 ; P=.006$ ).

\section{Tricuspid Valve Replacement}

A total of 8 patients $(2.4 \%)$ received TV replacement with a Medtronic Mosaic bioprosthetic porcine valve. All but 1 patient $(87.5 \%)$ who received TV replacement had severe TR. None of the patients with replacement received an RVAD. Prosthetic valve failure did not develop in any patients during the follow-up of 678 days. 


\section{DISCUSSION}

This study attempts to evaluate long-term durability and clinical impact of TVPs. The major findings are that (1) TV repair was durable at 2 years and prevented progression to significant TR and that (2) despite worse background characteristics, short- and long-term outcomes in patients received TVP were comparable to those in patients who did not receive TVP.

In this study, concomitant TVP was associated with increasing early morbidities, including prolonged $\mathrm{CPB}$ time, increased intraoperative platelets use, and higher incidence of bleeding requiring reoperation. These results were consistent with a previous large cohort study showing that TVP was associated with an increased risk for postoperative renal failure, greater total transfusion requirement, and longer hospital length of stay. ${ }^{3}$ The most probable cause for increased major morbidities is underlying preoperative progressive RHF in patients with significant TR. The TVP group clearly showed elevated $\mathrm{CVP}, \mathrm{CVP} /$ pulmonary capillary wedge pressure ratio, and total bilirubin. Also, $37.1 \%$ of the TVP group had severe $\mathrm{RV}$ dysfunction on preoperative echocardiography. Underlying RHF can lead to congestive hepatopathy and impair the synthesis of clotting factors, thus increasing the risk of bleeding. ${ }^{10}$ Another contributing factor would be risk of bleeding from additional atriotomy and prolonged $\mathrm{CPB}$ time. In our cohort, CPB time was increased by an average of 52.1 minutes after addition of TVP and was similar to what was reported in a meta-analysis by Dunlay and colleagues. ${ }^{4}$ However, in the TVP group, in-hospital mortality was $10.5 \%$ and 30 -day mortality was $3.7 \%$ in our series, which were comparable to previous series. ${ }^{11}$ More important, despite worse preoperative status in this cohort, these mortality rates were comparable to those in patients who did not receive TVP. RVAD was indicated in patients who showed severe RHF after LVAD implantation with a reported incidence of $5 \%$ to $9 \% .{ }^{12-14}$ Our study had a similar use of RVAD in the entire cohort $(7.9 \%)$ and a slight but nonsignificant increase in the TVP group $(13.2 \%)$. Piacentino and colleagues ${ }^{5}$ reported that TVP had less RHF measured by use of RVAD or more than 14 days of inotropes. Although the relationship between TVP and RVAD use is yet to be determined, TVP can be performed without any additional increase in the risk of being on RVAD support. Because we always perform TVP on a beating heart, there might be a decreased risk of RVAD use and early mortality associated with prolonged crossclamp time. From these results, patients with CF-LVADs should not be excluded from concomitant TVP because of the perceived increased operative risk.

Echocardiography results show the immediate and continuous benefit of TVP on reducing TR. In the TV repair group, $90 \%$ had no or mild TR within 1 month after initial discharge. In the long-term, longitudinal analysis of our data showed that TVP is protective against progression to significant TR up to 2 years on-device. This shows that TV repair has exceptional long-term durability. In our series, most patients $(\mathrm{n}=66 ; 97.1 \%)$ received ring annuloplasty, which might contribute to this favorable outcome. ${ }^{15}$ So far, some studies have reported that LVAD alone can improve the severity of TR, mostly by reducing RV afterload as CF-LVAD unloads the left ventricle., ${ }^{76-18}$ However, our results suggest that RV afterload does not explain the entire pathophysiology behind RV dysfunction and TR after prolonged LVAD support in all patients. In untreated patients with no significant TR, 34.7\% eventually progressed to significant TR. The results show that absence of significant TR intraoperatively does not imply that the tricuspid valve is structurally and functionally normal, because the degree of TR often changes by the loading condition. Moreover, patients with LVADs commonly have implantable cardioverter defibrillator leads leading to an anatomic cause for persistence of TR. Overall, a subset of patients with nonsignificant TR might benefit from TVP, although further studies are warranted to classify patients with the highest risk of developing TR in the long-term.

In this study, TV replacement was mostly reserved for patients with severe leaflet tethering or destruction. Our results show that TV replacement has excellent durability with no prosthetic valve failures seen during follow-up. Deo and colleagues ${ }^{19}$ showed that clinical outcomes, including the incidence of RHF, were unaffected by the decision to repair $(n=48)$ or replace $(n=16)$. Further studies with a greater number of patients are needed to evaluate the full benefits of TV replacement.

As more patients are supported longer on device, especially patients with destination therapy, long-term survival and functional outcome on device are essential in evaluating the efficacy of TVP. There have not been enough studies done on evaluating the long-term effects ( $>1$ year) of TVPs. ${ }^{4}$ One report from the HeartMate II trial showed 1 - and 2-year survivals of $77 \%$ and $63 \%$, respectively, in patients who received TVP. ${ }^{3}$ In our study, the TVP group mostly received the HeartMate II device $(\mathrm{n}=67 ; 88.2 \%)$ and long-term survivals were similar, with $75.2 \%$ and $63.7 \%$ survivals at 1 and 2 years, respectively. Furthermore, after 2 years of device support, long-term incidences of RHF readmission in patients with TVP are no different from patients with LVAD only. Again, patients in the TVP group did have preoperative signs of RHF as demonstrated by hemodynamic and laboratory variables. These factors are commonly associated with the development of postoperative RHF and poor long-term survival after CF-LVAD implantation. ${ }^{20,21}$ These results suggest the long-term benefits of concomitant TVP. Of note, subgroup analysis showed that patients with moderate TR but left untreated had worse on-device survival $(76.0 \%)$, whereas 
moderate TR treated with TVP showed comparable survival to nonsignificant TR $(93.3 \%$ vs $92.5 \%)$ (Figure 2). At the same time, patients with at least moderate-severe TR and treated with TVP had worse on-device survival compared with the nonsignificant TR group $(82.1 \%$ vs $92.5 \%)$. Thus, severe TR likely had a negative impact on survival even with repair, although a definitive conclusion cannot be drawn because of the lack of a true control group (patients with severe TR but untreated). This suggests that although repair is necessary for moderate or greater TR, earlier intervention before TR progresses to severe is needed to improve overall survival. Subgroup analysis of comparing TVP in patients with known moderate TR showed no difference in overall 6-month survival, although further statistical analysis cannot be performed because of the severely limited sample size.

\section{Study Limitations}

First, this was a retrospective study based on a singlecenter experience. However, the size of our cohort was larger than in most other centers, and we were able to be consistent in patient selection, surgical techniques, and medical management. Second, the number of patients in the TVP group was relatively low, which limits statistical power. However, this study has detailed follow-up data, including serial echocardiography results. Third, because many patients were censored at the time of transplant, the number of patients at 2 years is relatively small. Our study is one of the largest series to date, and most previous studies have focused on the immediate outcomes of TVP in LVAD implantation. Finally, regarding TVP, many surgical options exist, such as ring or suture annuloplasty, bioprosthetic valves, or mechanical valves. Our data could not clarify which procedure was more beneficial than others. Further prospective studies are warranted to carefully select patients who would benefit most from each technique.

\section{CONCLUSIONS}

TVP does have immediate adverse events, but most are reversible, so it can be performed safely in the short-term. TVP is durable at 2 years and may provide long-term clinical benefits in sick patients with significant TR. Therefore, patients receiving CF-LVADs with significant TR should be considered for concomitant TVP.

\section{Conflict of Interest Statement}

Y.N. received consulting fees from Thoratec Corp. All other authors have nothing to disclose with regard to commercial support.

\section{References}

1. Rose EA, Gelijns AC, Moskowitz AJ, Heitjan DF, Stevenson LW, Dembitsky W, et al. Long-term use of a left ventricular assist device for end-stage heart failure. N Engl J Med. 2001;345:1435-43.
2. Akhter SA, Salabat MR, Philip JL, Valeroso TB, Russo MJ, Rich JD, et al. Durability of De Vega tricuspid valve annuloplasty for severe tricuspid regurgitation during left ventricular assist device implantation. Ann Thorac Surg. 2014;98: 81-3.

3. Robertson JO, Grau-Sepulveda MV, Okada S, O'Brien SM, Matthew Brennan J, Shah AS, et al. Concomitant tricuspid valve surgery during implantation of continuous-flow left ventricular assist devices: a Society of Thoracic Surgeons database analysis. J Heart Lung Transplant. 2014;33:609-17.

4. Dunlay SM, Deo SV, Park SJ. Impact of tricuspid valve surgery at the time of left ventricular assist device insertion on postoperative outcomes. ASAIO J. 2015;61: 15-20.

5. Piacentino V, Ganapathi AM, Stafford-Smith M, Hsieh MK, Patel CB, Simeone AA, et al. Utility of concomitant tricuspid valve procedures for patients undergoing implantation of a continuous-flow left ventricular device. J Thorac Cardiovasc Surg. 2012;144:1217-21.

6. Saeed D, Kidambi T, Shalli S, Lapin B, Malaisrie SC, Lee R, et al. Tricuspid valve repair with left ventricular assist device implantation: is it warranted? $J$ Heart Lung Transplant. 2011;30:530-5.

7. Atluri P, Fairman AS, Macarthur JW, Goldstone AB, Cohen JE, Howard JL, et al Continuous flow left ventricular assist device implant significantly improves pulmonary hypertension, right ventricular contractility, and tricuspid valve competence. J Card Surg. 2013;28:770-5.

8. Nelson WB. Recurrent Events Data Analysis for Product Repairs, Disease Recurrences, and Other Applications. Philadelphia: SIAM; 2003.

9. Fitzmaurice GM, Laird NM, Ware JH. Applied Longitudinal Analysis. Hoboken, NJ: John Wiley \& Sons; 2004.

10. Matthews JC, Pagani FD, Haft JW, Koelling TM, Naftel DC, Aaronson KD. Model for end-stage liver disease score predicts left ventricular assist device operative transfusion requirements, morbidity, and mortality. Circulation 2010;121:214-20.

11. John R, Naka Y, Park SJ, Sai-Sudhakar C, Salerno C, Sundareswaran KS, et al Impact of concurrent surgical valve procedures in patients receiving continuous-flow devices. J Thorac Cardiovasc Surg. 2014;147:581-9.

12. Kormos RL, Teuteberg JJ, Pagani FD, Russell SD, John R, Miller LW, et al HeartMate II Clinical Investigators. Right ventricular failure in patients with the HeartMate II continuous-flow left ventricular assist device: incidence, risk factors, and effect on outcomes. J Thorac Cardiovasc Surg. 2010;139:1316-24.

13. Takeda K, Naka Y, Yang JA, Uriel N, Colombo PC, Jorde UP, et al. Outcome of unplanned right ventricular assist device support for severe right heart failure after implantable left ventricular assist device insertion. J Heart Lung Transplant. 2014:33:141-8.

14. Aissaoui N, Morshuis M, Schoenbrodt M, Hakim Meibodi K, Kizner L, Börgermann J, et al. Temporary right ventricular mechanical circulatory support for the management of right ventricular failure in critically ill patients. J Thorac Cardiovasc Surg. 2013;146:186-91.

15. McCarthy PM, Bhudia SK, Rajeswaran J, Hoercher KJ, Lytle BW, Cosgrove DM, et al. Tricuspid valve repair: durability and risk factors for failure. J Thorac Cardiovasc Surg. 2004;127:674-85.

16. Topilsky Y, Oh JK, Atchison FW, Shah DK, Bichara VM, Schirger JA, et al. Echocardiographic findings in stable outpatients with properly functioning HeartMate II left ventricular assist devices. J Am Soc Echocardiogr. 2011;24: 157-69.

17. Morgan JA, Paone G. Impact of reverse remodeling on cardiac function. Ann Cardiothorac Surg. 2014;3:589-94.

18. Lee S, Kamdar F, Madlon-Kay R, Boyle A, Colvin-Adams M, Pritzker M, et al Effects of the HeartMate II continuous-flow left ventricular assist device on right ventricular function. J Heart Lung Transplant. 2010;29:209-15.

19. Deo SV, Hasin T, Altarabsheh SE, McKellar SH, Shah IK, Durham L, et al. Concomitant tricuspid valve repair or replacement during left ventricular assist device implant demonstrates comparable outcomes in the long term. J Card Surg. 2012;27:760-6.

20. Kormos RL, Teuteberg JJ, Pagani FD, Russell SD, John R, Miller LW, et al. Right ventricular failure in patients with the HeartMate II continuous-flow left ventricular assist device: incidence, risk factors, and effect on outcomes. J Thorac Cardiovasc Surg. 2010;139:1316-24.

21. Kirklin JK, Naftel DC, Kormos RL, Stevenson LW, Pagani FD, Miller MA, et al Fifth INTERMACS annual report: risk factor analysis from more than 6,000 mechanical circulatory support patients. J Heart Lung Transplant. 2013;32:141-56.

Key Words: right heart failure, tricuspid valve, ventricular assist device 
TABLE E1. 95\% Confidence interval of survival function in Figure 1

\begin{tabular}{lcc}
\hline Days on support & Group A: TVP & Group B: No TVP \\
\hline 50 & $0.800-0.946$ & $0.902-0.963$ \\
100 & $0.766-0.926$ & $0.887-0.954$ \\
200 & $0.741-0.913$ & $0.838-0.922$ \\
300 & $0.646-0.866$ & $0.816-0.908$ \\
400 & $0.646-0.866$ & $0.767-0.877$ \\
500 & $0.587-0.843$ & $0.754-0.870$ \\
600 & $0.587-0.843$ & $0.712-0.845$ \\
700 & $0.587-0.843$ & $0.695-0.835$ \\
730 & $0.587-0.843$ & $0.676-0.825$ \\
\hline
\end{tabular}

TVP, Tricuspid valve procedure.

TABLE E2. 95\% Confidence interval of survival function in Figure 2

\begin{tabular}{lcccc}
\hline Days on support & a. Moderate TR + TVP & b. No significant TR + no TVP & c. Moderate-severe TR + TVP & d. Moderate TR + no TVP \\
\hline 30 & 1 & $0.939-0.989$ & $0.827-0.974$ & $0.810-0.987$ \\
60 & 1 & $0.912-0.976$ & $0.721-0.916$ & $0.710-0.943$ \\
90 & $0.613-0.990$ & $0.892-0.964$ & $0.721-0.916$ & $0.710-0.943$ \\
120 & $0.613-0.990$ & $0.892-0.964$ & $0.721-0.916$ & $0.710-0.943$ \\
150 & $0.613-0.990$ & $0.884-0.960$ & $0.692-0.900$ & $0.570-0.873$ \\
180 & $0.613-0.990$ & $0.876-0.955$ & $0.692-0.900$ & $0.570-0.873$ \\
\hline
\end{tabular}

$T R$, Tricuspid regurgitation; $T V P$, tricuspid valve procedure. 